\title{
Speaking with Things: \\ Encoded Researchers, Social Data, and Other Posthuman Concoctions
}

\begin{abstract}
We apply our heuristics for 'interviewing' nonhuman research participants (Adams and Thompson 2011) to the digital things of qualitative research itself: recording devices, data analysis software, and other sociomaterial concoctions recruited at different stages of contemporary research projects. We suggest that these 'inorganic organized' entities participate as co-researchers that inevitably extend but also disrupt research practice and knowledge construction, introducing new tensions and contradictions. Counterpointing phenomenology and Actor Network Theory, we usher some of the hidden and coded materialities of research practice into view, and glimpse unexpected realities coenacted. Such immersive entanglements raise ethical questions about the posthumanist fluencies now demanded in social science research practice and we outline several considerations.
\end{abstract}

Keywords: Actor Network Theory, coded materialities, interviewing objects, phenomenology, posthumanist fluencies, qualitative research, sociomateriality

Under the surface of our lives an increasingly complex geography of encoding is evolving with its own emergent performative outcomes-a performativity in which human agency is but a faint echo, silently shaping our present and future possibilities of becoming.

(Introna 2012, 114)

How can we hear, or give speech to, that which is silent?

(Ihde 1983, 69)

\section{Introduction}

Imagine a human or social scientist today without her familiar surround of $21^{\text {st }}$ century research tools: no laptop, no internet, no email, no digital recorder, no voice recognition or encryption software, no digitized transcripts or multimedia files, no data analysis software, not even a delete key! We may as well imagine a chef without knives, a consultant without a smartphone, a doctor without a stethoscope. The expertise of every professional is indebted and intimately tied to the ready availability of contemporary, specialized equipment in the performance of specialized work. In the context of the posthuman turn, researchers have begun to discern that the fluid assemblings of such equipment or 'inorganic organized' entities (Stiegler 1998) participate in co-constituting our skillful material enactments in the world. Of interest is not only how such thingly gatherings serve in the performance of professional practiceswhether cheffing, doctoring, or researching - but also the difference specific technologies make in the forming, informing, deforming, conforming, reforming, and transforming of practices and their performative outcomes.

The professional artifacts addressed in this paper are digital technologies - coded materialities - that are increasingly recruited for, enrolled in, and intertwined with human and social science research work. We draw on Actor Network Theory (ANT), phenomenology, and other approaches undergirding human-technology studies and posthuman theorizing to intervene, as Ruppert, Law, and Savage (2013) suggest, in ongoing debates about the digital as an epochal shift, by instead bringing attention to the specificities of the changes inaugurated by the digital. 
Posthumanism, a loosely associated set of perspectives, attempts to think beyond humanism, reconceptualizing the human not as an autonomous sovereign, but as an intimately entangled being inseparable from its technologies, environment, and other species. As Stiegler (1998) argues, we may no longer strive to separate the 'who' (anthropology) from the 'what' (technology), and instead we must grapple with the inscriptions of 'epiphylogenesis', i.e. the invention of the human via techne.

Yet we also know that in human and social science research, the co-constitutive material realm of everyday human life still tends to be overlooked as incidental or inconsequential rather than acknowledged and problematized. The aim of this article is threefold. First, we forward the notion that certain specialized objects - 'things' — contribute to research projects as coresearchers. To do this, we unravel several examples taken from empirical studies to explore the digital landscape of everyday qualitative research practices. This unraveling involved 'interviewing' one or more of the digital objects - cameras and recorders, iPods, data analysis software, and even the data itself - that were enrolled and entangled in each research project. The 'interview questions' employed are taken from eight heuristics we presented previously for engaging technologies-in-use as qualitative research participants (Adams and Thompson 2011).

Second, having illustrated our heuristics in action we briefly review the eight heuristics and add one more (gathering anecdotes). The heuristics, derived from our ANT (Thompson) and phenomenological (Adams) research work, offer a way for qualitative researchers to query the variety of materialities that may be holding sway in the research process. As we demonstrate, these heuristics may also be used to interview the digital things that researchers increasingly enlist in their own work. Finally, we consider the implications of including digital technologies as co-researchers and discuss the growing sophistication of digital fluencies now demanded of researchers. We begin with a brief consideration of how ANT and phenomenology may serve together as productive counterpoints on this posthumanist quest to query the contributions digital materialities make to research. Here we cite the work of Tim Ingold (2012), but others have uncovered similarly fruitful overlaps when combining insights from both approaches (cf. Introna 2007, Verbeek 2005).

\section{ANT and phenomenology, objects and things}

Ingold $(2012,435)$ contends that practitioners do not merely inter-act with their materials but rather co-respond with them: 'In the act of production, the artisan couples his own movements and gestures - indeed, his very life - with the becoming of his materials, joining with them and following the forces and flows that bring his work to fruition.' Drawing on Heidegger and Flusser, Ingold delineates an important phenomenological distinction between 'objects' and 'things'. An 'object', by definition, stands against us or is thrown in our path; at best we may interact with it. A 'thing', on the other hand, is materiality 'thinging'; it is

a gathering of materials in movement - a particular knotting together of the matter-flowand to witness a thing is to join with the processes of its ongoing formation. To touch it, or to observe it, is to bring the movements of our own being into close correspondence with those of its constituent materials. (436)

While Latour's human and nonhuman networked collectives helpfully serve to dismantle subject/object binaries, Ingold (2012) points out that crucial animating principles, such as human perception and response, are elided in Latour's 'skeletal' networks. Instead, Ingold offers SPIDER (Skilled Practice Involves Developmentally Embodied Responsiveness) as a means to 
draw the de-scriptive insights of actor network theorists and phenomenologists' reflective grasp of the prereflective lifeworld into a more productive, holistic dialogue: 'The emphasis in SPIDER is not on the interactive convocation of existing entities but on the co-responsive movement of occurrent things along their manifold lines of becoming' (437). Ingold proposes 'meshwork' as an enlivened reconceptualization of the ANT network, an interweaving of flow lines_-polyphonic melodies (von Uexküll 2010 [1934/1940])—emerging from the ongoing congress and play of living and non-living material forces.

Our own object interview questions or heuristics (Adams and Thompson 2011) similarly aim to reveal the 'thingly' enmeshments (Ingold 2012) of human and social science research through inviting Latour's (1992) 'missing masses' into the qualitative research interview to recall their everyday stories. Within an ANT framing, subject/object separations are undermined through an ontological demotion of the human (subject) and the promotion of the nonhuman (object) by recognizing agency as distributed. With phenomenology, subject/object boundaries are rendered translucent in the immediacy of the prereflective lifeworld. Phenomenology attends primarily to the pathic (passif) or perceptual sphere of human being-in-the-world, whereas ANT strives to describe the active (actif) world of humans and non-humans networked in dynamic assemblages. Practiced together, our heuristics embed and struggle with these two shifts in emphasis, creating actif-passif imbroglios for analysis and reflection. Via this collection of 'inventions', the researcher is in a position 'to make [objects] talk, that is, to offer descriptions of themselves, to produce, scripts of what they are making others - humans and non-humans - do' (Latour 2005, 79, emphasis in original), without killing the patient or pathic dimensions animating the human research site.

\section{Digital devices as co-researchers}

We now take a closer look at how social scientists may correspond with the flows, forces, and becoming of materials in their research practices. Re-reading anecdotes drawn from empirical studies that enrolled digital technologies in the performance of qualitative research projects, we employ several of our heuristics to interview these technologies-in-use - digital cameras and recorders, web artifacts, iPods, and Computer-Assisted Qualitative Data Analysis Software (CAQDAS).

\section{The unintended consequences of employing a digital co-researcher}

An educational researcher recalls an incident that occurred in the course of her qualitative research project investigating basic education in Western China (Crichton 2009, para. 8-9, abridged):

During a school visit in rural western Sichuan province, I wandered into the central playground. People in this region tend to be poor as the area is challenging to farm. It was lunchtime and the children were outside getting their lunches from the walls outside their rooms. I decided to film the children as they chatted happily. Sitting back down, I scrolled through the pictures I had just taken, ensuring that I had what I wanted, when I noticed a group of children just on the margins of one the images - these children were just outside the actual frame, but it was something about the freeze frame of the photograph that allowed me to notice something that I had not been able to observe when I watched the children earlier. I showed the picture to my colleague who explained, "Those children are pretending to eat lunch. Those children are too poor to bring food from home." Literally they were the children in the margins of more than my photograph; the ones who typically 
formed the large numbers of minority children who dropped out of rural schools after their compulsory Basic Education was completed - if not earlier. In an odd drama, these children sat off to the side, with their empty lunch boxes, pretending to eat, taking the same amount of time and effort as their classmates with actual food.

Crichton (2009) describes this incident as 'an unintended consequence' of doing digital documentation research. Such surprising occurrences reveal the unique but primarily taken-forgranted contributions that technologies, like the digital camera, make to current research practice. Indeed, it is the digital camera's specific capacity to both 'freeze frame' a windowed version of the lifeworld and then reproduce its collection of images on demand, that led to the unexpected noticing of the school children on the margins. Later, as Crichton (2009) relates, the digital photographs were used as 'evidence' of stark childhood poverty, upsetting local Chinese authorities' claims to the contrary. Here the digital camera evolves from a research instrument in the co-production of visual data, then to the co-construction and revelation of a politically contentious issue, next it shape shifts into evidence supporting a truth claim, then appears in a research manuscript as an anecdote, and finally, it shows up here in this journal article. We may speculate that the camera itself has now been replaced by a newer model, its research days done; the images may be locked in an encrypted file on Crichton's hard-drive, perhaps a few individual images also found in a PowerPoint presentation. Still, the camera's participation as an invaluable but unsung co-researcher lingers on in this recollected event.

In its taken-for-granted status as co-researcher, the digital camera acts as a visual documenter and archive, inviting the researcher to take multiple photographs, the opportunity to review these snapshots at the field site, and later, to transfer them to her research computer for storage, organization and import to any number of possible software. At the push button command of the principal investigator, the camera produces and stores 'frozen framings' of the lifeworld for later exposure and examination of the photographed research subject.

Via freezing, the research interest is arrested in its temporal tracks; the moment is focused, captured, and stilled. The subject is rendered 'objectively' available for closer scrutiny later as one of many pieces of visual data. Or as McLuhan (1964/2003) put it, the camera 'fix[es] people in a superior stare, as if they were objects' (257). The photograph as 'mass-produced merchandise' (and now as its fluid digital counterpart) is a 'brothel-without-walls' casting the individual into the public domain deprived of human privacy (257). More positively, the photograph is a 'museum without walls' (380): the captured visual image retrieves the past as present, a figure detached from its original ground. The snapshot directs attention to human posture and gesture, both individual and collective, while 'translat[ing] all senses into eye terms' (McLuhan and McLuhan 1988, 205). It promotes self-consciousness, and of course is deaf. The frozen fixity of the photographic image also creates 'a world of accelerated transcience' (McLuhan 1964/2003, 266), each found view is committed to an eternal stream of immobile ephemera. With the digital, the photographic stream has become thicker, more intense, and seeps into the filmic. With the digital, these immobilized images now circulate with newfound ease: at a press of a button or a swipe and click of a mouse, they are viewable, transferable, copy-able, share-able, crop-able, modifiable, delete-able.

Via framing, the lifeworld that falls outside the view-finder window disappears from the research scene; what falls inside - even along the edges of the frame - appears in a visually disclosive, evidentiary way and is collected as one piece of visual data. As a co-researcher, the camera 'enhances pictorial realism and obsolesces portrait painting' (McLuhan and McLuhan 
1988, 99), which serves to depict qualitative research as more a scientific ('objective') rather than aesthetic ('subjective') practice. Nonetheless, the human-researcher-with-digital-camera has become the curator of a specialized museum-without-walls, a museum whose collection is now closely monitored by human ethics boards worried by possible brothel-without-walls exposure. The digital complicates the patrol of these ethical borders, multiplying the opportunities for the photograph - the un-grounded figure - to fall into the wrong hands and be subject to the unforgiving eyes of the global village. Yet the very capacity of the photograph to 'expose' and thereby 'show' or 'prove' is also a primary reason for enlisting a digital camera in research practices. The human-researcher-with-digital-camera is able to generate a digitized, pictorial show, whose framings - and sometimes croppings or magnifications - may reveal previously unnoticed aspects of a world. Simultaneously, the research subject is immobilized as a visual data point, a file that is now available for immediate circulation, translation and transformation. The digital camera amplifies the critical, noticing eye, but may atrophy the ethical, relational eye.

Through employing a few of our object interview heuristics to interrogate the digital camera in situ - attending to its invitational address, studying an accident, then deploying McLuhans' Laws of Media while recognizing the amplification/reduction structure mobilized (see Appendix) - we have begun to discern how a technology may unexpectedly introduce new ethical tensions and responsibilities into research practice. Attributing co-researcher status to the camera helpfully brackets the humanist assumption of sole agency and reveals a more distributed, complex fold of enactments.

Whose, what's or who-what's research? As digital recording devices evolve beyond the camera and become more sophisticated, portable, and user friendly, they increasingly become skilled observers and listeners; not just of human research participants but also of other digital tracings. Gourlay (2012) describes a research project in which iPod Touch devices were distributed to students to document their everyday experiences of using technologies in their studies. Here, the iPod Touch takes on the role of field researcher standing in a close, $24 \times 7$ proximity to and juxtaposition with human participants. The artifacts generated by the digital device - photos, videos, sound files, and notes - are later called upon to act as data, but also as stimulus for more in-depth exploration in the face-to-face interviews. By beginning to follow the actors implicated in these practices (an ANT-inspired heuristic), one starts to see the interpellation of the iPod Touch and the digital artifacts as both participants (generating data through their presence and actions) and as co-researchers (storing and sharing data); a blurring of the positioning of objects and objects created by objects.

Also illustrated is what Ruppert, Law, and Savage (2013) describe as a de-centering of the human expert in order to elicit and generate data. Such implications of digital co-researchers have yet to be adequately theorized. Several tensions surface from this kind of de-centering and the pervasive, unblinking eye of the digital devices in the field. For example, more may be revealed than intended as these devices infiltrate private spaces and private moments, in the same way that Crichton's (2009) digital camera revealed unexpected nuances in the margins of digital photographs. Too, the digital data generated has a portability and potential 'social life' far beyond that of handwritten field notes, creating possibilities for further slippages and movements outside the traditional researcher-participant-data relationship. Finally, there could be both a deskilling and up-skilling of the researcher's work. Interfacing with digital co-researchers and working with the digital data generated now includes sophisticated digital curation skills: selecting, arranging, describing, annotating, aggregating (re)using, organizing, interpreting, 
storing, jettisoning, as well as the care for digital objects (i.e., Barrett 2012; Flanders and Munoz 2012).

\section{Making data gatherable: intercording}

Although the mundane and everyday provides rich opportunities to interview objects, much of the posthuman scholar's fieldwork is focused on multiplying the occasions of momentary visibility of objects. One helpful heuristic is to study accidents and breakdowns in order to reveal some of the intimate alliances which knit people and things together in everyday practices. The following anecdote, describing the process of data gathering, is reconstructed from a report of a disastrous interview episode by Michael (2004, 12-13):

I am conducting an interview with a respondent (Elle) at her home as part of a research project focused on public understanding of science. Seated on the sofa, Elle is in an armchair to my right, and the tape recorder placed on the floor between us. We begin. But what's this? Her pit bull dog has ambled into the room and sits on my feet! Moments later, her cat wanders in and starts playing with the recorder: clawing at it and pulling it along the ground by its strap - dragging it further and further away from Elle and me. Although being paid five pounds for the interview, Elle does nothing to intervene and instead goes on about her new job at Burger King. I cannot do anything to stop or redirect the conversation. I'm far too preoccupied with the disappearing tape recorder and the pit bull's liking for my feet.

Michael (2004) uses several strategies to untangle the role of non-human actors in this data collection interview. He begins by focusing on a breakdown, i.e., the apparent failure of a social science interview. Michael then constructs two co(a)gents - another heuristic - to explore the orderings and disorderings that took place among actors he follows and describes as 'the playful cat, the potential devil dog, the indifferent respondent, the petrified interviewer', the recorder, the 5 pounds, and other objects (14). He describes co(a)gents as analytical fabrications - 'delimited admixtures of the human and nonhuman' - which allow researchers to 'explore some of the complex heterogeneous interactions that make up social ordering processes' (10). In this manner, Michael examines the confrontation between the pitpercat (pit bull dog + person + cat) and the intercorder (interviewer + recorder). These two co(a)gents not only fail to interact as expected, the intercorder network simply falls apart as the recorder is dragged out of the room and the interviewer finds himself too distracted by everything going on to attend to the interview. There is a disruption, a disordering of relations. With the link between the interviewer and recorder becoming more tenuous as the interview proceeds, one effect of this weakened network is the absence of relevant data being produced or recorded.

Michael (2004) offers several insights in his analysis. First, the practice of making data gatherable is often enmeshed with technological and natural nonhumans, highlighting 'the heterogeneous disciplinary work that must be carried out in the production of social data' (1819). Second, he explores how sociological data is the effect of the orderings and disorderings within and between networks. We can do another reading of this anecdote drawing on other ANT concepts. To begin, nothing is quite as it seems. The interview is translated into an off-topic monologue, the interviewer is translated into a helpless bewildered bystander, and the recorder is translated into a cat's toy. Here, translation is a useful heuristic. Translations describe shifts and linkages (Law 2009a, 144). They are detours of sorts (Latour 1987); connections which transport 
transformations (Latour 2005). Law's (2009b) notion of collateral realities - the gaps and tensions between practices and their realities - helps to tease apart these translations. The explicit presence of the researcher, recorder, and the 5 pound payment alongside the implicit presence of the university and academic research protocols all fail to enroll the respondent in the expected way. The production and recording of useful and relevant data has been absenced. A very different reality is being enacted in the anecdote.

The fragility and tenuous nature of collecting data practices, and the data collection interview in particular, is now more visible. Law $(2007,126)$ points to the tensions within practices, commenting that if practices look streamlined then it is because the bits that do not fit and the choreography that holds it all together are simply not visible or understood. If an interview hangs together it is only a temporary assemblage, easily disrupted unless something happens to make it either more stable or more elastic and able to 'absorb' unexpected actors and actions. The presence of the interviewer and recorder is not enough. The complexity of the broader network in which the intercorder must become entangled - the sociality of making data gatherable (Michael 2004) - is reinforced by this micro-analysis. Although researchers are aware that sometimes practical and technical circumstances result in interviews which cannot be recorded, this analysis points to a more granular consideration of the dynamic ecology of human and nonhuman actors that must achieve some sense of spatial and temporal stability so that the effect is indeed the production of useful data.

Anecdotes as heterogeneous interlocutors. Thus far, several anecdotes have been presented and unraveled. But what is an anecdote and may it also serve as a heuristic to sharpen the sensitivities of a researcher to the thinging of research? More recently, Michael (2012) reflects on this episode, commenting that it is not merely an anecdote about methodological failure and 'lessons learned', but rather a revealing of the 'ways that social data are made possible by virtue of the disciplining (or silencing) of non-human others' (30). The performativity of this anecdote, particularly its irritant qualities, continued to work on him over the years and led to a rethinking of the episode. Michael introduces the notion of anecdotalization in which 'the making and enacting of anecdotes is a means of interrogating the research process itself' (33). Here, the performativity of the anecdote alerts the researcher to new sensitivities.

Notably, anecdotes, or petits récits, enable humans and nonhumans to show themselves circulating and unfolding through time and space with the fluidities (and stoppages) they enjoy 'in the wild'. As short plausible fictions that are gathered and reconstructed from field observations and interviews, anecdotes attempt to re-evoke living moments in which humans and things participate and have 'voice' in the world. Such 'descriptive constructions' (Hirchauer 2006, 436) provide a way to reconstruct and then unravel the complexity of the fluid, essencing lifeworld. Anecdotes unfold. As such, they may be considered a kind of epistemic object. Knorr Cetina $(2001,181)$ explains that epistemic objects are 'processes and projections rather than definitive things', characterized as 'open, question-generating and complex'. She adds that such 'objects of knowledge':

have the capacity to unfold indefinitely.... Since epistemic objects are always in the process of being materially defined, they continually acquire new properties and change the ones they have. But this also means that objects of knowledge can never be fully attained, that they are, if you wish, never quite themselves.

Never quite themselves, anecdotes enact and are enacted by the lifeworld that enabled them. They are multi-voiced and provide a way for things to speak as well as a way of speaking with things. Anecdotes are not simply 'data upon which we work' (Stronge and Michael 2012, 28). 
Rather, "the "flow" of anecdotization means that they end up as something akin to "heterogeneous interlocutors" in the inventive of doing research' (Michael 2012, 34).

\section{Freezing digital data: exclusion and inclusion}

Law and Urry (2002) posit that current social science methods deal poorly with the fleeting, distributed, multiple, non-linear, and chaotic. After all, how does one research the kind of fluidity and entanglement described here?

The Web is a process, happening continuously and continuously transforming before our eyes; with us and through us. Technologies appear and then dissolve in the peripheries, websites are built, they bloom and then pass away, but the Web continues, because we are the Web. (Czerski 2012, para. 13)

Wikis, blog postings, online forum discussions: such digital data or data collection spaces are in a state of constant transformation. As illustrated in the digital camera anecdote above, researchers are often implicated in practices of stabilization or freezing. In a web context this materializes as printing web pages, taking screen captures, copying and pasting text and images into a Word document, or sliding digital finds off into Evernote. And so, the cast of characters grows more complex and entangled. Following these actors, questions arise about what it is that is being archived; or in Latour's (2005) words, what is happening downstream. Kallinikos, Aaltonen, and Marton (2010) argue that such snapshots are pages that have been temporarily assembled and rendered in a particular space and time; no longer dynamic web pages but static ones whose link to original sources which update the page now severed. These digital artifacts are now longer mobile but frozen.

Practically, such practices enable researchers to cope with the volume of information continually propagated by the web. Cairns and Birchall (2013) suggest that the horizontal organization of the internet is mobilizing "new tactics, both algorithmic and social, to help make sense and meaning from the swaths of hyperconnected, hyperflexible data" (para. 7). However, there are other implications. If the web is an indissoluble weaving of human-technology actors, as Czerski (2012) suggests, then a screen capture is perhaps also enacting what Law (2009b) refers to as a barely noticed collateral reality; one of boundary making. Kallinikos, Aaltonen, and Marton (2010) describe digital objects as unbounded, evasive, distributed, and constantly mutating. Therefore, they suggest that such archiving practices do not simply collect already bounded entities but rather 'construct the boundaries that demarcate and make an archival document' (7). Through everyday acts of freezing digital objects, researchers alongside their technological co-researchers, are very much engaged in translation of digital artifacts and data, excluding and including along the way. Following actors inevitably leads to decisions not to follow certain actors. Suchman $(2007,284)$ describes cutting the network as 'a practical, analytical and/or political act of boundary making'. She explains that methodologically, one predicament is to decide how any object of analysis - human or nonhuman or a combination - is extricated from the more extended networks of which it is part.

Is anything lost when dynamic web pages are translated into static data? This is difficult to answer because any form of data collection, digital or otherwise, is an act of 'freezing' of sorts. Implications to be considered include acknowledging what has been excluded - where the network has been cut. Law (2004) urges researchers to attend to the messiness of othering. He points out that 'matters are relational: what is being made and gathered is in a mediated relation with whatever is absent, manifesting a part while Othering most of it' (146). 
How technologies interpose data within the research process - freezing, thawing, excluding, including - beckons researchers to attend to the sociality of data. In moving to a posthuman perspective, data - a blackboxed materiality of research projects - becomes much more complex. In attempting to gather data, understand it, and interpret it, data is necessarily lashed together with many other things, most especially encoding technologies. Yet, data is not really a thing but rather a relational effect: it is what it is in a particular moment because of the temporal and spatial networks of relations in which it is ensnared. Indeed, Ingold $(2012,431)$ argues for less focus on the 'objectness' of things and more attention to the 'material flows and formative processes wherein they come into being'. We believe that data is not something pointed to as a concrete bounded object shuttling from one point in the research process to the next, but rather is a continual re-enactment of social and material relations. Cole (2008) presents three competing conceptualizations of data: as commodity, as purified, and as differentiated. The third conceptualization sees data as de-centered, heterogeneous, and hybrid. He argues that the data-concept standing alone cannot hold; coherence is achieved and maintained within ongoing conversations and processes of negotiation and exchange. It seems data is an assemblage - and a fluid one at that.

The way that data both enacts and is enacted within research practices highlights the ongoing tension between fluidity of data (the state of always becoming and creating ongoing movements in understanding) and solidification of data (freezing or the act of settling down and settling into a particular locality). Freezing data, at certain points, into an artifact gives it a momentary shape and presence so it can then be shaken into something else in a different set of relations. So that it can open up new meanings, uncertainties, understandings, disruptions. This is not a linear process. Data can enact multiple realities simultaneously. In ANT terminology, data could be considered both an immutable and mutable mobile (interesting distinctions to tease apart when following the actors). Latour (1990) describes an immutable mobile as an object which maintains its form thereby fixing ideas and practices in place so that they can circulate and mobilise other networks.

In contrast, Law and Singleton (2005) describe mutable mobiles - fluid objects - as defined by a set of relations that gradually shifts rather than holding itself rigid. It is possible that data may even be what Law and Singleton term fire objects: messy objects that jump and are discontinuous; different and yet partially connected. Objects which flicker in and out of presence and absence. Multiplicities. The notion of data as a fire object is amplified when we consider digital data. Indeed, Ruppert, Law, and Savage (2013) refer to the 'liveliness' of digital data. What is digital data? It can take many forms: a Word document, numbers in an online database, online artifacts, or algorithms coded into software. Because of its liveliness it is not confined to one space or time, juxtaposed with other things in limitless ways, shifting and adapting as it is entangled in other networks. Even when it is frozen.

\section{The encoding of researchers}

We now return to our opening paragraph, where we imagined a researcher bereft of her usual assortment of research tools. QSR International, the makers of NVivo qualitative research software, recently constructed a similar imaginary in a YouTube video (2012a), portraying their software as a 'solution', presumably to the 'problem' we call qualitative inquiry. The following is an excerpt from that video:

If you don't use software...working manually can...make it very hard to discover subtle connections in your data. NVivo gives you a place to organize and manage your material 
[at this point, the video shows four "hand-drawn" filing cabinets labeled Sources, Themes, Analysis, and Findings] so that you can start to make sense of your data. It also provides tools that allow you to ask questions of your data in a more efficient way.... take stock of what your data is saying about [your research topic] all in the one place. Finally, this all might come together as a report or a presentation.

QSR International intends to situate NVivo at the core of every qualitative research project, from start to finish: interview data 'in', research report 'out'. Yet this software architecture rests on a series of unexamined design decisions that may,

configure and circumscribe us...in more or less significant ways, defining what is relevant and what is not, what needs to be attending to and what not-legitimating particular ways of being whilst simultaneously delegitimizing (or rendering more or less obscure) equally valid alternatives. (Introna 2012, 115)

In order to reveal the 'more or less significant ways' NVivo may serve to re-configure, recircumscribe, and redefine research practice, several 'object interview' questions may be posed: What specific activities does NVivo software invite a researcher to perform? What epistemological frameworks are implicitly or explicitly mobilized in this virtual work environment? And what practices are discouraged, delegitimized or merely diminished?

To explore NVivo's invitational or pathic address, imagine a researcher who has decided to use NVivo. Entering the NVivo 10 software workspace, she is immediately prompted to create a New Project or Open an existing one. Choosing New Project, the researcher is then invited to select from one of multiple tabs including import 'External Data', 'Analyze', 'Query', and 'Explore'. Under the Query tab, she may choose such activities (commands) as 'Text Search', 'Word Frequency', and 'Coding'. Under the 'External Data' tab, the researcher is asked to import data in any of the following digital formats: 'Documents, PDFs, Datasets, Audios, Videos, Pictures, and Memos'. Non-digitized data (written field notes) as well as unsupported digital formats (a PowerPoint presentation) may be accounted for, if not 'imported', as 'Externals' under 'From Other Sources'. She begins by importing all his digital content so far, which consists of several transcribed interviews.

Consulting the 'Getting Started Guide', the researcher reads she may now begin by 'broadbrush' coding via the creation of thematic 'Nodes' or by jumping straight into detailed coding (QSR International 2012b, 23). She may, for example, perform 'in vivo ' coding' by selecting a resonant phrase in a transcript, then clicking on 'Code In Vivo' in the 'Quick Coding' bar. NVivo also offers an 'Auto Code' feature, which will automatically code structured datasets such as transcribed interviews that employ identical questions. The researcher decides to jump straight into 'detailed coding' of a transcript, since this appears to most closely correspond with how she previously approached her qualitative analysis work by hand. Quickly, the qualitative researcher finds herself immersed in the NVivo workspace, busily coding and querying data.

Necessarily overlooked in this productive human-technology engagement is how the NVivo software code is simultaneously and prereflectively encoding the researcher, both hermeneutically and existentially. All such software provides clearly delineated, finite sets of activities, along with a specialized, local vocabulary designed to ease user navigation of the software environment and the performance of allowable tasks, and an implicit database structure

\footnotetext{
${ }^{1}$ The term in vivo - Latin for 'within the living'-is more commonly used in microbiology to distinguish between different types of experimentation with living cells and organisms (cf. ex vivo and in vitro), and can be traced back to Glaser \& Strauss' (1967) grounded theory.
} 
scaffolding how the researcher may accomplish data analysis work. In qualitative inquiry, the term code generally refers to 'a word or short phrase that symbolically assigns a summative, salient, essence-capturing, and/or evocative attribute for a portion of language-based or visual data' (Saldaña 2013, 3). The verb form, to code, is an activity performed on data, 'an interpretive act' that variously 'summarize [s], distill[s], or condense [s] data, not simply reduce [s] them' (4, italics in original). In NVivo, the noun form of code is not used at all, but is replaced with the word Node (helpfully rhyming with code); the verb form, to code, now unambiguously understood as a function that the researcher performs by creating uniquely named (coded) Nodes, which may be hierarchically arranged as tree structures. In NVivo, coding is synonymous with the creation of Nodes that may later be queried. Outside qualitative research circles, coding is more commonly associated with programmers (e.g. designing, writing and testing computer code or programs) and cryptographers (e.g. deciphering a code or encoding secret messages). Importantly, concepts such as codes, nodes, queries, and encryption belonged to computing science and database theory long before qualitative inquiry met NVivo. Is it possible that in aligning their scholarly practices with NVivo, researchers are quietly being reconfigured as specialized database administrators?

During a phenomenological research project investigating compassion fatigue among health professionals, Goble, Austin, Larsen, Kreizer and Brintnell (2012) were struck by the difference using Computer-Assisted Qualitative Data Analysis Software (CAQDAS) seemed to make to their research practices. In an effort to understand some of these effects, they employed another of our object interview heuristics, the McLuhan's (1988) four laws of media. Media theorist Marshall McLuhan and his son Eric proposed that every technology obeys four 'Laws of Media', which are held in a dynamic fourfold structure. They posed these laws - enhancement, obsolescence, retrieval, and reversal - as questions intended to reveal the totality of an artifact's medial effects on the larger socio-cultural environment:

- What does [this technology or medium] enhance or intensify?

- What does it render obsolete or displace?

- What does it retrieve that was previously obsolesced?

- What does it produce or become when pressed to an extreme? (McLuhan and McLuhan $1988,7)$

Applying these laws, Goble et al. (2012) composed the following tetrad. CAQDAS, and more specifically NVivo:

- Enhances 'the equality of data in data sifting';

- Obsolesces 'the tactility of research' including the disappearance of handwritten notes and playing with cut-up transcripts;

- Retrieves 'the punch-card expert'; and when over-used it

- Reverses into 'data shuffling'.

Goble et al. $(2012,12)$ noticed that, on the one hand, the data analysis software served their team in 'effectively remov[ing] irrelevant sections of transcripts while bringing forth the relevant sections...so none would be overlooked.' On the other hand, the core researchers simultaneously became distanced from the original texts, no longer working with it 'by hand'. Data entry, a new but relatively unskilled task demanded by CAQDAS, was easily 'outsourced' to research assistants. Finally, the research team struggled with the activity of coding central to CAQDAS: [W] hen coding becomes the means by which analysis takes place and supersedes ones methodology, only generalized thematic description becomes possible. For compassion 
fatigue researchers, the appeal of coding risked their never exploring the phenomenon's essence. (Goble et al. 2012, 13)

Having committed their study to NVivo, they unexpectedly found their deeper methodology compromised. CAQDAS enhances the agency of the qualitative researcher. This agential extension is enacted via the researcher's subscription to the methodological assumptions and design decisions made by its manufacturer. In order to use CAQDAS, the researcher must, to some extent, adjust their practices to accommodate those adumbrated by the software algorithm. In response, the researcher's habits of mind shift and reconfigure, gestural regimes topple and reconstitute, familiar vocabularies take on new softwared significances, and everyday modes of knowing, thinking and doing undergo subtle and occasionally dramatic changes.

\section{Interviewing the coded materialities of research practices}

In this paper we have sought to give the things of qualitative research — digital recorders and cameras, multimedia files and web artifacts, iPods and qualitative data analysis software-a voice. To accomplish this, we employed a variety of heuristics or 'specific tricks' to make the digital materialities of research practice talk, 'that is, to offer descriptions of themselves, to produce scripts of what they are making others-humans or non-humans - do' (Latour 2005, 79). In a previous article (Adams and Thompson 2011) we presented eight heuristics for interviewing objects in an effort to bring matter out of the background and into critical inquiry. These heuristics emerged in the context of our own qualitative research projects-one phenomenological (Adams 2006; 2010; 2012), the other ANT-based (Thompson 2012a; $2012 b$ ) - as we struggled to articulate the unique contributions specific digital technologies make across different teaching and learning contexts - formal and informal; face-to-face and online. As we experimented with different ways to encourage things to speak to us, we realized that we had admitted these technologies as nonhuman research participants. We referred to the etymological origins of the word 'interview':

It is derived from the old French verbal noun s'entrevoir, composed of two parts: entre-, meaning mutual or between, and voir, to see, which together mean "to see each other, visit each other briefly, have a glimpse of". Thus to "interview an educational artifact" is to catch insightful glimpses of the artifact in action, as it performs and mediates the gestures and understandings of its employer, involves others, and associates with other objects (Adams and Thompson 2011, 734).

The object interview heuristics were proposed as starting points for exploration, much like a protocol designed for semi-structured research interviews. Each heuristic provides a different sensitivity for recovering nonhuman contributions at the research site. Not all need to be applied, not all questions asked. By employing a composition of ANT- and phenomenology-based queries, overlaps as well as contradictions are expected. In the previous section, we have tried to illustrate how our heuristics might be employed to examine the 'meshwork' of research practice. We now pull together and briefly outline the heuristics. We have added one more: gathering anecdotes. We have also separated these nine heuristics into two clusters of inquiry: one focused on educating the researcher's attention to things, the other on analyzing materialities of practice. For a more detailed rendering of the heuristics, refer to Adams and Thompson (2011), as well as to the list of suggested 'interview' questions in the Appendix.

\section{Attending to objects, attuning to things}


The first cluster of heuristics provides possible entry points for attending and attuning to the digital technologies that matter to research and professional practice. We include here: gathering anecdotes, following the actors, listening for the invitational quality of things, and studying breakdowns and accidents.

Gathering anecdotes. At the heart of our gesture to interview objects is the desire to give things a voice in human and social science research. Yet, it is often difficult to hear and convey the voices of things. Indeed, object voices do not translate easily into the quote format popular in qualitative reports. We have come to appreciate how gathering, constructing and mobilizing anecdotes has both a generative and disruptive impact on the inclusion of things as legitimate coresearchers and participants. Michael $(2000,14)$ explains that the anecdote 'allows one to start from an incident and trace out a range of associations'. In this way, these local petits récits serve as entry points to further analysis of the materialities of research practice.

Following the actors. The point of 'following the actors' is to look for 'mediators making other mediators do things', human or non-human (Latour 2005, 217). Each of the anecdotes above brings an array of actors into view, as well as questions about the multiple and at times, collateral realities, being enacted. Although an object, such as an iPod Touch, provides an entry point for a researcher, ANT theorists focus on the 'connected' object or as Bruni $(2005,358)$ describes, the 'relational game in which objects are involved (and which objects themselves activate)'. It is the sociality around the object that creates an entry point for the researcher. To follow the actors, the researcher must listen for the invitational quality of things.

Listening for the invitational or 'pathic' quality of things. From a phenomenological perspective, the things of our immediate world speak to us prereflectively. My digital camera invites me to take multiple photographs at a research site, nVivo promises to assist me in my work as a qualitative researcher-organizing, analyzing and reporting. Of course I may ignore all these appellations. But having responded to the 'call' of a thing, I am swiftly caught up in the particular world that it opens: snapping photos or importing and coding my digital data. Articulating such thingly invitations provides a glimpse of the lines of force that are set in motion each time we involve a technology in our practice. These 'default" lines serve to delineate, support and enhance some practices while diminishing, dissolving or even thwarting others.

Studying breakdowns and accidents. Like Michael's (2004) calamitous interview, and Crichton's (2009) unintended digital framings, breakdowns and accidents offer rich grounds for making the work of objects and things visible. As Latour $(1992,229)$ remarks in his analysis of a door, 'every time you want to know what a non-human does, simply imagine what other humans or other non-humans would have to do were this character not present'.

\section{Loosening the meshwork, analyzing digital materialities}

Having attuned to material presences and absences, an array of other heuristics may help to analyze - to gently loosen - a digital artifact's meshwork in order to reveal otherwise hidden aspects of its thingly participation and contribution. We include here: discerning the spectrum of human-technology relations, recognizing the amplification/reduction structure of humantechnology relations, applying the 'laws of media', unravelling translations, and constructing co(a)gents.

Discerning the spectrum of human-technology relations. While not applied in this paper, post-phenomenologist's Don Ihde's (1990) human-technology relations-embodiment, hermeneutic, alterity, and background - can to alert us to the multiple ways we engage 
technologies everyday, as well as to how these intimate relational engagements silently reshape practice both existentially and hermeneutically.

Recognizing the amplification/reduction structure of human-technology relations. All human-technology relations display an amplification/reduction structure. On the one hand, a technology extends or amplifies a particular human capacity. On the other hand, it also reduces, diminishes or hobbles another capacity. Every technology has what Ihde $(1983,56)$ calls 'a telos, or weighted center of gravity which makes it partially selective as to what may be enhanced and what reduced'. They are Janus-faced (Arnold 2003). For example, we saw that the digital camera enhances fluidity and public accessibility while simultaneously eroding fixity and diminishing individual privacy.

Applying the 'Laws of Media'. Goble et al. (2012) provide an excellent example of how the McLuhan's Laws of Media may be used to query a technology-in-use, yielding insight into how a technology simultaneously enhances and disrupts, and ultimately reshapes current practices in sometimes unexpected ways.

Unraveling translations. As a researcher unravels multiple entangled actor-networks, all sorts of tensions, contradictions, and uncertainties will emerge. Translation (explained earlier), an ANT concept, enables researchers to look at how collections of entities come to be aligned or co-constituted with other actors and networks in a particular practice. It is through translations that entities interface with others, transform, become linked or de-coupled. And so, in the Crichton (2009) anecdote, a simple example of translation is how the production of a digital image is transformed from visual data into evidence and ultimately into an anecdote in a research manuscript.

Constructing co(a)gents. Illustrated by Michael's $(2004,10)$ analysis of the disastrous interview episode was the fabrication and use of co(a)gents, such as the pitpercat and the intercorder, to 'examine and explicate relations, connections, and interactions that are barely apparent but nevertheless serve in the (de)structuring of everyday routines'. In a similar fashion, phenomenological understanding supersedes the classical separation of subject and object. The lifeworld is characterized by the transpermeation (Rosen 2006) and intermingling of subject(s) and object(s) through the 'reciprocal insertion and intertwining of one in the other' (MerleauPonty 1968, 138).

These heuristics are intended to heighten qualitative data collection and analysis sensitivities. They may also enable researchers to keep a critical eye on the sociomaterial enactments of the things they themselves have enlisted in their research projects. Yet, we remind that these heuristics are tentative, themselves materialities which can translate and betray as Mol (2010, 265-266), writing from an ANT perspective, explains:

The terms and texts that circulate in ANT are co-ordination devices. They move topics and concerns from one context to another. They translate and betray what they help to analyse. They sharpen the sensitivity of their readers, attuning them/us to what is going on and to what changes, here, there, elsewhere. In one way or another they also intervene, not from a place of overview, but rather in a doctoring mode. They care, they tinker.

\section{Concluding remarks: speaking with things}

A complex churn of organic and inorganic entities contributes to the enactment of research and performance of data. Social scientists increasingly perceive, think, act, and speak with and through their digital things. In recognizing our hybrid, networked, posthuman selves, the invisible, silent nonhuman research crew supporting our efforts suddenly becomes apparent. Like 
our human research assistants, it now seems important that we take time to interview our nonhuman counterparts before we set to work together as a team. Michael $(2004,5)$ asks: 'how might we go about theorizing the role of nonhumans (technologies, animals, etc.), and their associations with humans, in the production of social data?' We outlined nine heuristics that may help researchers attend to the role of thingly gatherings of research practices.

Ingold $(2012,434)$ writes that 'to understand materials is to be able to tell their historiesof what they do and what happens to them when treated in particular ways - in the very practice of working with them'. This is a way of speaking with things. As our agency as researchers is increasing shared, distributed and supported by digital materialities, new ethical-political considerations come into play. As Hirschauer (2006) argues, technologies like recording devices have served to depreciate 'homemade' ethnographic description in favour of 'accurate' transcriptions, endangering sociology's claim to 'make a difference'. The retreat of this complex 'cultural technique' from ethnographic research practice, an observational writing activity honed only in the face of forgetfulness, is symptomatic of the rise of digital 'mnemotechnologies' currently destabilizing institutions (Stiegler 2011). Enrolling the latest technologies into one's research projects may seem an ethically sound practice, but a closer examination suggests a more complex picture.

Through counterpointing ANT and phenomenology, we have begun to bring the invisible things of research into view and acknowledge the often unexpected realities enacted incidentally throughout research practices. Such immersive entanglements raise questions about the posthumanist fluencies now demanded in social science research practice. Gourlay $(2011,1)$ describes posthumanist literacies as "practices of meaning-making in a context where the boundaries between analogue and digital, "human" and "machine", are ambiguous and problematic'. Our focus in this paper is on those fluencies that matter when researchers become entangled with digital technologies as co-researchers. We prefer the phrase fluencies to literacies, since it connotes a stronger sense of responsibility, expertise, and criticality in one's humantechnology interactions and correspondences.

Our analysis suggests, as a starting point, that such fluencies could include first, interviewing objects as co-researchers to unpick the politics of objects and uncover the ethics of things as they stand in for and work alongside the researcher, all the while co-defining the processes and products of research. Secondly, the ability of researchers to navigate the blurred boundaries of encoded practices, including the translation of qualitative research practices into digital curation and database management, is becoming more significant. Finally, the ostensible mobility of the digital itself, and the "capacity for the circulation, sharing and take up of devices and data across numerous sites' (Ruppert, Law, and Savage 2013,39) suggests that knowledge making and knowledge spaces are becoming more lively, distributed, public, and fragmented as web and mobile technologies, including social media, become more embedded in everyday research practices. The scale, mobility, and spatial arrangements in which data unfolds are being radically configured. Such energies call for further exploration. 


\section{References}

Adams, C. 2006. PowerPoint, habits of mind, and classroom culture. Journal of Curriculum Studies 38, no. 4: 389-411.

Adams, C. 2010. Teachers building dwelling thinking with slideware. The Indo-Pacific Journal of Phenomenology 10, no. 2: 1-12.

Adams, C. 2012. Technology as teacher: Digital media and the re-schooling of everyday life. Existential Analysis 23, no. 2: 262-273.

Adams, C., and T. L. Thompson. 2011. Interviewing objects: Including educational technologies as qualitative research participants. International Journal of Qualitative Studies in Education 24, no.6: 733-50.

Arnold, M. 2003. On the phenomenology of technology: the "Janus-faces" of mobile phones. Information and Organization 13: 231-56.

Barrett, K. 2012. Object lessons - curating in the 21st century. http://spoonsontrays.blogspot.ca/2012/09/object-lessons-curating-in-21stcentury.html\#!/2012/09/object-lessons-curating-in-21st-century.html

Bruni, A. 2005. Shadowing software and clinical records: On the ethnography of non-humans and heterogeneous contexts. Organization 12: 357-78.

Cairns, S., and D. Birchall. 2013. Curating the digital world: Past preconceptions, present problems, possible futures. http://mw2013.museumsandtheweb.com/paper/curating-thedigital-world-past-preconceptions-present-problems-possible-futures/

Cole, F.T.H. 2008. Taking "data” (as a topic): The working policies of indifference, purification and differentiation. Paper presented at the $19^{\text {th }}$ Australasian Conference on Information Systems, Christchurch, New Zealand. ACIS 2008 Proceedings. Paper 79. http://aisel.aisnet.org/acis2008/79

Crichton, S. 2009. A picture is worth a thousand meals: digital documentation and unintended consequences in development work. Unpublished manuscript.

Czerski, P. 2012. We, the web kids. Trans. M. Szreder. http://pastebin.com/0xXV8k7k.

Flanders, J., and T. Muñoz. 2012. An introduction to humanities data curation. http://guide.dhcuration.org/intro/

Glaser, B. G. \& Strauss, A. L. (1967). Discovery of grounded theory: Strategies for qualitative research. Hawthorne, NY: Aldine de Gruyter.

Goble, E., Austin, W., Larsen, D., Kreitzer, L., \& Brintnell, E. 2012. Habits of mind and the split-mind effect: When computer-assisted qualitative data analysis software is used in phenomenological research. Forum Qualitative Sozialforschung / Forum: Qualitative Social Research. [Online] 13:2. http://www.qualitativeresearch.net/index.php/fqs/article/view/1709

Gourlay, L. 2011. Cyborg literacies and the posthuman text. Paper presented at the Workshop on New Media, New Literacies, and New Forms of Learning, December. http://blogs.ubc.ca/newliteracies/files/2011/12/Gourlay.pdf

Gourlay, L. 2012. Student author as actor network? Using ANT to explore digital literacies in higher education. In Proceedings of the 8th International Conference on Networked Learning 2012, ed. V. Hodgson, C. Jones, M. de Laat, D. McConnell, T. Ryberg, and P. Sloep, 97-102. http://www.networkedlearningconference.org.uk/info/confpapers.html

Heidegger, M. 1971. The origin of the work of art. Poetry, Language, Thought. Trans. A. Hofstadter. NY: Harper Collins. 
Hirschauer, S. 2006. Putting things into words. Ethnographic description and the silence of the social. Human Studies 29: 413-41.

Ihde, Don. 1990. Technology and the lifeworld: from garden to earth. Bloomington: Indiana University Press.

Ingold, Tim. (2012). Toward an ecology of materials. Annual Review of Anthropology 41: 42742.

Introna, L. 2007. Maintaining the reversibility of foldings: Making the ethics (politics) of information technology visible. Ethics and Information Technology 9, no. 1: 11-25.

Introna, L.D. 2011. The enframing of code: Agency, originality and the plagiarist. Theory, Culture and Society 28, no. 6: 113-41.

Kallanikos, J., A. Aaltonen, and A. Marton. 2010. A theory of digital objects. First Monday 15, no. 6-7. http://www.firstmonday.org/

Knorr Cetina, K. 2001. Objectual practice. In The practice turn in contemporary theory, ed. T.R. Schatzki, K. Knorr Cetina, and E. von Savigny, 175-88. London: Routledge.

Latour, B. 1990. Drawing things together. In Representation in scientific practice, ed. M. Lynch and S. Woolgar, 19-68. Cambridge, MA: The MIT Press.

Latour, B. 1992. Where are the missing masses? A sociology of a few mundane artifacts. In Shaping technology/building society: Studies in sociotechnical change, ed. W.E. Bijker and J. Law, 225-58. Cambridge, MA: MIT Press.

Latour, Bruno. 1987. Science in action: How to follow scientists and engineers through society. Cambridge, MA: Harvard University Press.

Latour, Bruno. 2005. Reassembling the social: An introduction to actor-network theory. Oxford: Oxford University Press.

Law, John. 2004. After method: Mess in social science research. Abingdon, UK: Routledge.

Law, J. 2007. Pinboards and books: Juxtaposing, learning, and materiality. In Education and technology: Critical perspectives, possible futures, ed. D.W. Kritt and L.T. Winegar, 125-49. Lanham, MD: Lexington Books.

Law, J. 2009a. Actor network theory and material semiotics. In The new Blackwell companion to social theory, ed. B. S. Turner, 141-58. Chichester, UK: Wiley-Blackwell.

Law, J. 2009b. Collateral realties. http://heterogeneities.net/publications/Law2009CollateralRealities.pdf

Law, J., and J. Urry. 2002. Enacting the social. http://www.lancs.ac.uk/fass/sociology/papers/law-urry-enacting-the-social.pdf

Law, J., and V. Singleton. 2005. Object lessons. Organization 12, no.3: 331-55.

McLuhan, M. (1964/2003). Understanding media: the extensions of man, (critical edition), ed., W. T. Gordon. Corte Madera: Ginko Press.

McLuhan, Marshall, and Eric McLuhan. 1988. Laws of media: the new science. Toronto, ON: University of Toronto Press.

Michael, M. 2004. On making data social: Heterogeneity in sociological practice. Qualitative Research 4, no. 1: 5-23.

Michael, M. 2012. Anecdote. In Inventive methods: the happening of the social, ed. C. Lury and N. Wakeford, 25-35. Abingdon, Oxon: Routledge.

Michael, Mike. 2000. Reconnecting culture, technology and nature: From society to heterogeneity. London: Routledge.

Mol, A. 2010. Actor-network theory: sensitive terms and enduring tensions. Kölner Zeitschrift für Soziologie and Sozialpsychologie 50, no. 1: 253-69. 
QSR International. 2012a. Introducing NVivo (YouTube video). http://www.youtube.com/watch?v=7bLZ7fqSEEc

QSR International. 2012b. NVivo 10 Getting Started Guide. http://download.qsrinternational.com/Document/NVivo10/NVivo10-Getting-StartedGuide.pdf

Ruppert, E., J. Law, and M. Savage. 2013. Reassembling social science methods: The challenge of digital devices. Theory, Culture \& Society 30, no. 1: 22-46.

Saldaña, J. 2013. The coding manual for qualitative researchers. $2^{\text {nd }}$ ed. Thousand Oaks, CA: Sage.

Stiegler, Bernard. 1998. Technics and time 1: The fault of Epimetheus. Stanford, CA: Stanford University Press.

Stiegler, Bernard. 2011. Technics and time, 3: Cinematic time and the question of malaise. Trans. S. Barker. Stanford, CA: Stanford University Press.

Stronge, P. and M. Michael. 2012. Suggestion and satisfaction: on the actual occasion of agency. In Agency without actors? New approaches to collective action, eds. J-H. Passock, B. Peuker, and M. Schillmeier, 15-30. Abingdon, Oxon: Routledge.

Suchman, Lucy. 2007. Human-machine reconfigurations: Plans and situated actions. 2nd ed. Cambridge: Cambridge University Press.

Thompson, T. L. 2012a. I'm deleting as fast as I can: Negotiating learning practices in cyberspace. Pedagogy, Culture \& Society 20, no. 1: 91-110.

Thompson, T. L. 2012b. (Re/Dis)assembing learning practices online with fluid objects and spaces. Studies in Continuing Education 34, no. 3: 251-66.

Verbeek, Peter-Paul. 2005. What things do: philosophical reflections on technology, agency, and design. Cambridge, Mass: MIT Press.

Von Uexküll, Jakob. 2010 [1934/1940]. A foray into the worlds of animals and humans; with, A theory of meaning. Trans. J. D. O’Neil. Minneapolis, MN: University of Minnesota Press. 


\section{Appendix \\ Interview Questions for Digital Objects and Other Things}

Attending to objects, attuning to things

Gathering anecdotes

Anecdotes are short plausible fictions that are gathered and reconstructed from field observations and interviews with human research participants.

- Describe how the object or thing appeared, showed up, or was given in an everyday life situation or practice. What happened?

Following the actors

As one follows the actors, it is important to attend to what is being mobilized and enacted (knowledge, beliefs, or actions) through the visible and the invisible interactions of actors.

- How does this particular assemblage come to be? How are people and objects brought into proximity with each other? How does this assemblage interact with other multiple networks?

- How did it come to be configured this way? What gets 'related' to what and how?

- What was disrupted or stabilized to create this particular configuration?

- What does this assemblage enact? What collateral (Law 2009b) realities are being enacted?

Listening for the invitational quality of things

- What is a particular technology inviting (or encouraging, inciting or even insisting) its user to do?

- What kind of epistemological or hermeneutic scaffolding is this technology tacitly offering its user to help frame his or her thinking or to amplify perceptual abilities?

- What is this technology implicitly or explicitly discouraging (or constraining, or even prohibiting) its user from knowing, doing or thinking?

Studying breakdowns and accidents

- Did anything unexpected happen when this technology was used? Were there any unintended or surprising consequences?

- What if a particular object was not there or what if it did not work?

Loosening the meshwork, analyzing digital materialities

Discerning the spectrum of human-technology relations

- What kinds of human-technology relations does this technology engage?

- Embodiment? Hermeneutic? Alterity? Background? (Ihde 1990) Others?

Recognizing the amplification/reduction structure of human-technology relations

- What human capacity is extended, enhanced or amplified when this technology is used?

- What is diminished, attenuated or simply 'forgotten' or laid aside?

Applying the 'Laws of Media'

- What does [this technology or medium] enhance or intensify? 
- What does it render obsolete or displace?

- What does it retrieve that was previously obsolesced?

- What does it produce or become when pressed to an extreme? (McLuhan and McLuhan $1988,7)$

\section{Unraveling translations}

- What is the choreography of a particular practice?

- What are the tensions, contradictions, uncertainties?

- How is it that networks, the entities enacting these networks, and/or the connections between networks, interface with other actor-networks in acts of transformation, linking up, or uncoupling?

- If an actor-network seems stable how did that stability transpire? If an actor-network seems to be in a state of flux, what is keeping it in a state of flux? Do some actornetworks seem more powerful or persuasive than others? If so, how have the specificities of that particular juxtaposition come to be?

- What is entrenched? What is excluded? What is made present? absent?

Constructing co(a)gents

Try (re)attaching conceptually the human(s) to the nonhuman(s) in play, treating the assemblage as a single corps, and ask:

- What enactments are rendered possible in this organic-inorganic imbroglio?

- What tensions and ambiguities live in the hyphened relational spaces conjoining the human and the nonhuman? 